\title{
De la violence à la non-violence Une lecture girardienne des "Juifves" de Robert Garnier
}

\section{Bernard Gallina}

\section{(2) OpenEdition}

1 Journals

Édition électronique

URL : http://journals.openedition.org/studifrancesi/6153

DOI : 10.4000/studifrancesi.6153

ISSN : 2421-5856

Éditeur

Rosenberg \& Sellier

\section{Édition imprimée}

Date de publication : 1 novembre 2010

Pagination : $429-441$

ISSN : 0039-2944

\section{Référence électronique}

Bernard Gallina, « De la violence à la non-violence Une lecture girardienne des "Juifves" de Robert Garnier », Studi Francesi [En ligne], 162 (LIV | III) | 2010, mis en ligne le 30 novembre 2015, consulté le 13 janvier 2021. URL : http://journals.openedition.org/studifrancesi/6153 ; DOI : https://doi.org/ 10.4000/studifrancesi.6153

\section{(c) (i) $\odot$}

Studi Francesi è distribuita con Licenza Creative Commons Attribuzione - Non commerciale - Non opere derivate 4.0 Internazionale. 


\section{De la violence à la non-violence \\ Une lecture girardienne des "Juifves" de Robert Garnier}

Celuy qui entreprend d'estre plus qu'il ne peut,

Souvent, trompé d'espoir, dechet plus qu'il ne veut.

(Les Juifves, II, vv. 205-206).

Cette recherche a pour objet le problème de la violence, de ses sources et de ses prolongements dans Les Juifves de Robert Garnier ${ }^{1}$. Ce thème parcourt constamment la tragédie, et il est sans cesse alimenté par des sèmes renvoyant à la cruauté, au sang, à la mort; Raymond Lebègue relève que la scène où s'affrontent les deux antagonistes, Nabuchodonosor et Sédécie, atteint une violence qui demeure inégalée dans le théâtre du XVI ${ }^{\mathrm{e}}$ siècle ${ }^{2}$. Michel Jeanneret affirme que ce théâtre est hanté par la violence et la terreur ${ }^{3}$. La rivalité mimétique, le déchaînement des passions, la recherche du bouc émissaire plongent ce texte dans «l'ordre du sacrificiel», pour adopter des termes chers à René Girard; en outre, l'intertexte biblique constitue la source profonde de la tragédie de Robert Garnier ainsi que de l'œuvre girardienne. C'est à partir de celle-ci, en nous appuyant sur elle, que nous entreprendrons notre approche critique.

\section{La rivalité mimétique}

Dès l'incipit, le prophète, médiateur entre Dieu et les hommes, lance un acte d'accusation contre le peuple élu, qui n'a pas suivi la route indiquée par l'Eternel, qui a même remplacé ce dernier par des idoles créées à son image, semblable en cela aux peuples qui n'ont pas entendu l'appel de Yaveh:

Helas!voyla que c'est d'offenser l'Eternel,

Qui te portoit, Sion, un amour paternel:

Tu as laissé sa voye, \& d'une ame rebelle

Preferé les faux Dieux qu'adore l'Infidelle.

Ingrate nation, tu as sur les hauts lieux

Osé sacrifier à la Royne des Cieux,

Luy consacrer des bois, tu as d'argille molle

(1) L'édition princeps de cette tragédie remonte à 1583: Les Juifves, tragédie de R.G.P., Paris, par P. Patisson, $1583,12^{\circ}, 32 \mathrm{ff}$. Cette œuvre fut ensuite publiée dans l'édition complète des tragédies en 1585: Les tragédies de R.G.P., Paris, par P. Patisson, $12^{\circ}$, XII-332 ff. L'édition de référence est la suivante: R. GARNIER, Les Juifves, Édition critique, établie, présentée et annotée par S. LARDON, Paris,
Honoré Champion éditeur, 1999.

(2) Cf. Notice, dans: R. Garnier, Les Juifves; Hippolyte, texte établi et présenté par R. LEBĖGUE, Paris, Les Belles Lettres, 2003, p. 115.

(3) Préface, dans: R. Garnier, Les Juifves, édition présentée, établie et annotée par M. JEANNERET, Paris, Gallimard, 2007, p. 7. 
Poitrie entre tes mains façonné mainte Idole,

Que tu as adoree. (abominable fait!)

Immolant à un Dieu, que toy mesme t'es fait. (I, vv. 67-76)

S'il abandonne la voie indiquée par le Très Haut pour atteindre la plénitude, l'homme ne renonce pas à l'être que constitue son modèle, car tout désir est désir d'être, avance en substance René Girard ${ }^{4}$, et il est infini. L'homme essaie d'atteindre Dieu en créant des divinités, en les idolâtrant pour imiter, singer le culte qui lui est dû. L'essence du désir métaphysique est soumise à une médiation, et présente un caractère triangulaire; elle est externe si le médiateur est hors d'atteinte, et interne si le médiateur est à la portée du sujet. Alors qu'il gît en prison en compagnie du pontife Sarrée, Sédécie lui confesse qu'il a été victime d'un médiateur externe, lorsqu'à l'instar de son peuple il est tombé dans l'idolâtrie, à laquelle il attribue son malheur:

C'est pour avoir peché devant ta sainte face,

O père, \& n'avoir craint le son de ta menace:

Te reputant semblable à ces Dieux que lon fond,

Ou qu'en pierre \& en bois les statuaires font,

Qui n'ont ame ny force, abominable ouvrage,

Aux hommes abestis qui leur vont faire hommage. (IV, vv. 1287-1292)

Plus loin, il relève également le rôle néfaste du médiateur interne lorsqu'il déclare:

J'ai failli, j'ai péché, j'ay suivi les sentiers

Des Rois, qui reprouvez m’ont été devanciers. (IV, vv. 1293-1294)

Le médiateur interne s'incarne dans les rois rejetés avant l'avènement au trône de Sédécie. Parmi eux, on peut citer sans doute son frère Joachas (Yoakhaz); son demi-frère, le fils de Josias et de la reine Zebida, Elyaqim à qui le roi d'Egypte donna le nom de Joachim (Yoyaqim); le fils de celui-ci, Yoyakîn. Tous commirent le péché d'idolâtrie; les deux derniers se révoltèrent contre Nabuchodonosor, qui riposta en ordonnant la première déportation du peuple juif sous le règne de Yoyakîn.

On peut supposer que pour le roi de Jérusalem, le médiateur interne s'incarne également dans le roi d'Assyrie. Ce dernier est en effet l'homme le plus puissant de la terre, il domine le reste des humains par son omnipotence, il s'impose à eux: il matérialise un degré de puissance, d'autonomie qui ne peut que susciter l'envie, la jalousie des autres hommes, et en particulier de Sédécie, qui essaie de recouvrer des possessions ayant appartenu à son royaume. Naît ainsi une mimésis, une rivalité d'appropriation avec le plus grand monarque de la terre. La mimésis-rivalité d'appropriation prélude à une mimésis-rivalité de l'antagoniste. Comme le dit René Girard à ce propos,

Plus les rivalités s'exaspèrent, plus les rivaux tendent à oublier les objets qui en principe les causent, plus ils sont fascinés les uns par les autres. La rivalité se purifie de tous ses enjeux extérieurs, en somme, elle se fait rivalité pure ou prestige. Chaque rival devient pour l'autre le modèle-obstacle adorable et haïssable, celui qu'il faut à la fois abattre et absorber5.

(4) R. GIRARD, Quand ces choses commenceront, entretiens avec M. Treguer, Paris, Arléa, 1994, p. 28.
(5) Des choses cachées depuis la fondation du monde, recherches avec J.-M. Oughourlian et G. LEForT, Paris, Grasset, 1978, p. 34. 
Quant à Nabuchodonosor, quelque puissant qu'il soit, il est incapable de se suffire, de se complaire à lui-même: il doit s'appuyer sur un objet externe pour tenter d'apaiser son désir métaphysique. Il n'échappe pas, lui non plus, à la médiation: il accuse l'influence du médiateur externe, lorsqu'il commence à se comparer à des médiateurs divinités de la mythologie latine, à rivaliser avec eux:

Pareil aux Dieux je marche, \& depuis le réveil

Du Soleil blondissant jusques à son sommeil,

Nul ne se parangonne à ma grandeur Royale,

En puissance \& en biens Jupiter seul m'égale:

Et encore n'estoit qu'il commande immortel,

Qu'il tient un foudre en main dont le coup est mortel,

Que son thrône est plus haut, \& qu'on ne le peut joindre,

Quelque grand Dieu qu'il soit, je ne suis pas moindre. (II, vv.181-188)

Ou bien lorsqu'il se risque à revendiquer la place qu'occupe le Dieu des monothéistes, à l'évincer de celle-ci:

Je suis l'unique Dieu de la terre où nous sommes. (II, v. 192)

Il proclame sa suprématie sur tous les monarques et les peuples de la terre, sur tous ceux qu'il parvient à atteindre, accusant ainsi l'influence qu'exercent sur lui les médiateurs internes:

Le Parthe m'obeist, le Persan \& le Mede,

Les Bactres, les Indois, \& cet Hebrieu cuidoit,

Rebelle, s'affranchir du tribut qu'il me doit. (II, vv. 200-202)

Nabuchodonosor et Sédécie apparaissent liés par une rivalité mimétique où l'un vise à s'imposer à l'autre, alors que ce dernier essaie d'échapper à ce dessein: le rival devient ainsi un obstacle à la réalisation du désir métaphysique, à la volonté d'être.

\section{Le maître, l'esclave, et l'escalade de la violence}

Dans l'une des premières scènes de la tragédie, Nabuchodonosor énonce l'un des principes de sa conduite à Nabuzardan:

Le service des miens soigneux je remunere. (II, v. 267)

S'installant dans la position du deus ex machina de la situation, de possesseur de l'univers, il affirme qu'il récompense ceux qui l'entourent d'attention, qui lui rendent service: apparaît ainsi la dialectique du supérieur et de l'inférieur, du maître et du valet.

Son attitude à l'égard d'Amital diverge profondément de celle qu'il adopte à l'encontre du fils de celle-ci. La voyant prosternée à ses pieds, il lui dit:

Levez-vous, je ne veux pas que vous soyez ainsi. (III, v. 1009)

Et il saisit l'occasion pour évoquer l'affection qu'il éprouvait pour son mari:

J'ai tousjours bien aimé Josie vostre espoux. (III, v. 1110) 
Plus loin, il réaffirme son estime, sa gratitude pour le feu roi, qui est mort sur le champ de bataille de Mageddon (Meguiddo) pour le soutenir dans sa lutte contre le roi d'Egypte: en un mot qui a reconnu en lui son souverain, son maître:

Quand ressemblant Josie un prince Judaïque

N'a prins pour moi le parti Memphitique,

Je l'ay gratifié l'assistant au besoing,

Et les bornes jetant de ses terres plus loin. (III, vv.1163-1166)

Josie (Josias) a répondu à l'attente qu'avait placée en lui Nabuchodonosor, reconnaître en lui son roi, être un vassal fidèle. Amital rappelle à Nabuchodonosor que sa famille a payé lourdement son engagement à ses côtés dans la lutte contre Nechun (Néko): non seulement son mari a donné sa vie pour son souverain, mais leur fils aîné Joachas qui s'est opposé lui aussi au pharaon a été emprisonné en Egypte sur l'ordre de ce dernier ${ }^{6}$. Et elle fait allusion au tribut en argent et en or que dut payer son pays au vainqueur:

A ce esté quand mon fils lié comme un forçaire

Fut esclave pour vous, sa ville tributaire? (III, vv. 1176-1177)

Or, contrairement à son père et à son frère, le second fils de Josie et d'Amital, Sédécie, après qu'il a été imposé sur le trône à la place de Yoyakîn, rétabli dans les droits liés à son appartenance à la branche aînée de la descendance de Josias par Nabuchodonosor $^{7}$, trahit son souverain pour opérer un renversement d'alliance en prenant le parti de Nechun. Le roi d'Assyrie entame un authentique réquisitoire lorsqu'Amital le supplie de pardonner sa trahison à Sédécie:

Vous ne parliez pas ainsi, quand en fiere arrogance

Vos enfants rebellez despitoyent ma puissance,

Amorcez du secours dont l'Egypte a manqué

Car alors sans raison vous m'avez attaqué. (III, vv. 1035-1038) celui-ci:

Etablissant un parallèle entre son mari et son fils, il lui reproche la rébellion de

Qui a son fils émeu de s'armer contre moi? (III, v. 1121)

Dans cet échange verbal, il énonce en raccourci ses chefs d'accusation contre Sédécie et les siens: complicité avec l'ennemi, rébellion contre son autorité, manquement à la parole donnée, mépris de sa puissance et de lui-même. Aux attaques politiques se surimposent chez Nabuchodonosor les griefs de caractère personnel. Il révèle les passions qui l'agitent dans sa conversation avec Nabuzardan:

Ce brave me pensoit si failli de courage,

De souffrir m'estre fait un si vilain outrage,

Et ne m'en ressentir, n'avoir point la raison

D'une si detestable et lasche trahison. (II, vv. 207-210)

(6) Cf. Deuxième Livre des Rois, 23-25.

(7) Yoyakîn était le fils de Yoyaqim, fils de Josias et de Zevidda. Ce fut le pharaon qui après avoir changé son nom d'Elyaqim en Yoyaqim, l'établit come roi, après avoir mis au chaînes son demi-frère Joachas. Cf. Deuxième Livre des Rois, 31-35. 
Et surtout il lui annonce son intention de rabaisser son rival, de le châtier pour sa tentative d'affranchissement:

Ce sont les grands qu'on doit punir plus griefvement. (II, v. 286)

Profondément blessé dans son orgueil, il savoure la capture de celui qui a essayé de contester sa suprématie, et il le ravale au rang d'un animal sauvage, d'une proie que l'on chasse avec furie:

Je le tiens je le tiens, je tiens la beste prise,

Je jouis maintenant du plaisir de ma prise,

J'ai chassé de tel heur que rien n'est eschappé:

J'ai lesse et marquacins ensemble enveloppé. (III, vv. 887-890)

Au moment où il s'apprête à affronter Sédécie dans un duel dont on entrevoit l'acharnement et la violence, Nabuchodonosor s'est installé dans la position du mầtre face à un esclave pris en flagrant délit de rébellion; dans celle du vainqueur qui condamne le vaincu à passer sous les fourches caudines; dans celle de juge et partie, car à lui revient le pouvoir de décision dans une affaire où il est personnellement impliqué. Quant à Sédécie, il doit à la foi endosser les conséquences de sa révolte et les responsabilités d'avocat de la défense pour lui et pour son peuple. Assumant les fonctions d'avocat général, de ministère public, Nabuchodonosor accumule les chefs d'accusation contre le roi de Jérusalem et sa suite, qu'il apostrophe avec véhémence:

Hà rustres je vous tiens,

Vous estes à la fin tombez en mes liens.

Toi, méchant déloyal, le pire de la terre,

$\mathrm{Tu}$ as induit ton peuple à me faire la guerre

Après t'avoir fait Roy, t'avoir au throne mis

De ton père, \& pour toi avoir les justes Rois démis?

Homme ingrat \& parjure, abominable Prince,

Tu as donc pour loyer revolté ma province?

Est-ce ainsi, malheureux, que tu me reconnois?

Est-ce ainsi que tu rens le bien que tu reçois? (IV, vv. 1373-1382)

S’il n'épargne pas dans son réquisitoire les partisans de Sédécie, c'est surtout contre ce dernier qu'il lance ses imputations, imputations à caractère moins politique que personnel. Il l'accuse moins de rébellion que de perfidie, et d'ingratitude: il lui rappelle ouvertement qu'il n'a pas hésité à destituer le roi Yoyakîn pour lui permettre d'accéder au trône.

Emporté par sa haine, «Celui que je hay tant», confie-t-il à Nabuzardan en parlant de son adversaire (II, v. 230), le Roi d'Assyrie s'en prend aux fondements de la foi de Sédécie et de son peuple, qu'il tourne en dérision:

Qui t'a mis en l'esprit de faulser ta parole?

N'en faire non plus cas que de chose frivole?

De parjurer ta foy? seroit-ce point ton Dieu,

Ton Dieu, qui n'a credit qu'entre le peuple Hebrieu?

N'est.ce point ce Pontife, \& ces braves Prophetes,

Les choses predisans après qu'elles sont faites?

Respons traistre, respons, où t'es-tu confié

De guerroyer celui qui t'a gratifié? (IV, vv. 1383-1390) 
Le roi de Jérusalem riposte en affirmant la toute-puissance de son Dieu, en récitant un acte d'humilité:

Le Dieu que nous servons est le seul Dieu du monde,

Qui de rien a basti le ciel, la terre \& l'onde:

C'est luy seul qui commande à la guerre, aux assaus:

Il n'y a Dieu que luy, tous les autres sont faux. (IV, vv. 1391-1394)

Il réhabilite la figure des prophètes en montrant leur rôle éminent de porte-parole, leur capacité de révéler les vérités cachées grâce à l'inspiration divine,

Ses Prophetes il a, que par fois il envoye

Pour radresser son peuple alors qu'il se devoye:

Par eux de nos malheurs il nous fait advertir,

A fin qu'en l'invoquant les puissions divertir. (IV, vv. 1399-1402)

Il rétorque au roi d'Assyrie qu'il ne doit sa victoire sur les Juifs qu'à la volonté de Dieu, qui veut infliger une punition au peuple élu:

soyez seur que toute force humaine,

Quand il nous est propice, encontre nous est vaine

Et qu'encor vos soudars, bien qu'ils soyent indomtez,

Ne nous eussent jamais comme ils ont surmontez,

Sans qu'il a retiré de nous sa bien-veillance,

Pour nous faire tomber dessous vostre puissance. (IV, vv. 1407-1412).

Plus loin, il l'invite à prendre son Dieu en exemple, à ne pas adopter une attitude tyrannique.

Si Sédécie s'avoue coupable,

Or vous ay-je offensé, je confesse ce poinct,

Je vous ay offensé: mais qui n'offense point? (IV, vv. 1413-1414)

il demande néanmoins à Nabuchodonosor d'user d'indulgence à son égard,

Le devoir vous défend de m'estre trop severe. (IV, v. 1432)

compte tenu de son ascendance royale,

J'estois auparavant fils \& frère de Roy. (IV, v. 1437).

Il refuse d'être humilié par Nabuchodonosor, ce qui donne naissance à une série d'accrochages verbaux de grande intensité:

SÉDÉCIE Je suis prince issu de sang royal.

nABUCHODOnOSOR Tu es Prince voir'ment, mais Prince desloyal. (IV, vv. 1441-1442)

SÉDÉCIE Votre honneur est de veincre \& savoir pardonner.

NABUCHODONOSOR Mon honneur est de veincre \& de reguerdonner. (IV, vv. 1449-1450)

Lorsqu'il comprend que son sort est scellé, qu'il n'échappera pas à la vengeance du roi d'Assyrie, il essaie dans une ultime tentative d'obtenir au moins le pardon pour son peuple: 
Vous estes en ce lieu,

Le temple, la vertu, la semblance de Dieu,

N'exercez dessur nous un pouvoir tyrannique,

Ains sauvez pour le moins cette tourbe Hebraïque. (IV, vv. 1465-1468)

Constatant que Nabuchodonosor demeure inflexible dans sa décision d'étancher sa soif de vengeance non seulement sur lui mais encore sur le peuple élu, il laisse éclater sa fureur passant brusquement du vouvoiement au tutoiement, en le souillant même lorsqu'il lui dénie toute origine nobiliaire, et pis encore toute aristocratie de l'esprit:

Sus donc cruel Tyran, assouvi ton courage,

Enivre toi de sang, rempli toi de carnage:

La bourreau ne te lasse, infecte l'air de corps,

Egorge les enfans, tire le cœur des morts,

Et le mange affamé, developant ta rage

Pire que d'un lion \& d'une tygre sauvage.

Tu n'as le cœur royal, \& aussi n'es-tu

Sorti de noble race, ains d'un lignage bas,

De la fange d'un peuple, \& d'une main brigande,

As couru l'Assyrie, où ta fureur commande. (IV, vv. 1479-1488)

Le combat redouble d'intensité, la violence croît entre les deux bretteurs qui finissent par s'insulter:

NABUCHODONOSOR Tu sembles un mâtin, qui abaye \& qui grongne.

SÉDÉCIE C'est toy-mesmes mâtin, qui te paîs de charongne,

NABUCHODONOSOR Empoignez-le, soudars, \& le tirez d'icy,

Je ne tarderai guere à le rendre adouci.

SÉDÉCIE Cherche nouveaux tourmens, \& sur moy les deploye,

Consulte tes bourreaux, tout cela ne m'effroye.

NABUCHODONOSOR Le desespoir qu'il a le rend audacieux,

Ou bien pour m'emouvoir il fait le furieux;

Mais son effort est vain, il ne sçauroit tant faire

Qu'il evite sa peine, elle est exemplaire. (IV, vv. 1495-1504)

Les métaphores, et en particulier les métaphores animales assénées à l'adversaire; la fréquence des mots renvoyant à la souffrance, au sang, à la mort; la présence de la stichomythie, qui est «l'équivalent des coups alternés que se portent deux adversaires dans le combat singulier», pour adopter les termes de René Girard ${ }^{8}$, indiquent la violence de l'affrontement qui met aux prises les deux rois. Le roi d'Assyrie abandonne le combat, la joute oratoire en ne parvenant plus à trouver, à porter une botte pour parer celle de l'adversaire. Il ne peut qu'ordonner à ses soldats de se saisir de son adversaire, que proférer de nouvelles menaces à son encontre. Le roi de Jérusalem parvient quant à lui à adresser un nouveau défi en lui lançant qu'il ne le craint point, mais il se rend compte que son plaidoyer se solde par un cuisant échec, puisqu'il n'a réussi ni à se sauver lui-même ni à sauver son peuple. Il refuse de reconnaître l'omnipotence du roi d'Assyrie, de le considérer comme l'incarnation de la puissance, même sous la forme dégradée de la tyrannie; qui plus est, il contre-attaque en magnifiant Dieu, le seul être tout-puissant à ses yeux, en stigmatisant et la conduite et l'origine obscure de 
son adversaire, qui à son avis est indigne de porter le nom de roi. Il ne lui renvoie pas l'image que celui-ci attendait de lui, même pas celle d'un esclave en proie à la terreur devant son maître: privé de la médiation qui lui permettrait de se sentir «l'unique Dieu de la terre» (v. 192), comme il se définit lui-même, Nabuchodonosor ne parvient pas à assouvir son désir métaphysique. Avide du regard de l'autre, dépendant de ce désir, il a attendu en vain le tribut, la reconnaissance, la gratitude de celui qu'il considérait désormais comme son esclave, finissant à son tour par devenir l'esclave de ce dernier. Dans cette humiliation réciproque, le maître s'est ainsi transformé en esclave de son esclave, et l'esclave en maître de son maître; victoire à la Pyrrhus, car l'esclave en proie à son orgueil échoue dans sa tentative d'obtenir de son maître le pardon pour lui et pour son peuple ${ }^{9}$, redevenant ainsi son esclave; lui non plus n'a pas obtenu le signe, la marque de reconnaissance qu'il sollicitait. Au lieu d'atteindre leur but, les flèches se sont retournées contre les archers. Dans cette mêlée chaotique, les deux adversaires tendent à perdre leurs différences, les hiérarchies à s'effacer, le sens à faire défaut: on reconnaît là l'émergence de la dé-différenciation, de l'indifférenciation. Or, comme le dit René Girard, c'est l'indifférenciation qui engendre la violence, c'est-à-dire «toute activité destructrice capable d'anéantir la vie et de l'appauvrir et de l'humilier ${ }^{10}$. Cet élément nouveau qu'est l'indifférenciation provoque la violence, de la même manière que la variation dans la tension de la corde d'un violon crée le désaccord, la discorde:

Take but degree away, unture that string

And hark what discord follows!,

affirme Ulysse dans Troilus and Cressida ${ }^{11}$. N'ayant pas réussi dans son face à face oratoire à l'emporter sur Sédécie, accusant l'effacement des signes qui le distinguent de son adversaire, Nabuchodonosor va donner libre cours à sa brutalité pour le briser, l'annihiler, ce qui provoque chez lui une escalade de la violence. Il annonce à Nabuzardan sa résolution de punir son adversaire:

Mais devant que le jour ait sa course finie,

Je jure qu'il verra sa lascheté punie. (II, vv. 211-212)

Lorsque son lieutenant l'exhorte à prendre en pitié son adversaire, alléguant sa condition de roi tombé en esclavage, il laisse éclater sa rancune:

Pour cela n'est encor ma vengence assouvie. (II, v. 223)

Assouvir sa vengeance devient la ligne inspiratrice de sa conduite, l'idée-force de celle-ci. Il renchérit en avançant que:

Qui tient son adversaire,

Et ne le meurtrit point, n'est vengé qu’à demy. (II, vv. 225-226)

Il ne cesse d'associer dans son esprit l'idée de punition et celle de vengeance, de les afficher. Il résiste aux supplications de la reine; celle-ci, profondément consciente du démon qui s'est emparé de son mari, confirmée dans ses convictions par son entrevue avec Amital, essaie de le fléchir, de lui arracher le pardon pour Sédécie:

(9) Sabine Lardon voit dans la maladresse et surtout dans l'orgueil de Sédécie l'explication de son désastre. Cf. Introduction, op. cit., pp. 3031.
(10) Article «Violence», dans Encyclopédie Philosophique Universelle, Paris, P.U.F., 1985.

(11) W. Shakespeare, Troilus and Cressida, Sc. 3. The Greek camp. Before Agamemnon's tent. 
LA ROYNE La douceur est tousjours l'ornement d'un monarque.

NABUCHODONOSOR La vengence tousjours un brave cœur remarque.

LA ROYNE Rien ne le souille tant qu'un fait de cruauté.

NABUCHODONOSOR Qui n'est cruel n'est digne de royauté. (III, vv. 908-910)

À la fin de cette rencontre, il lui concède qu'il laissera la vie sauve à son adversaire, sans renoncer cependant à lui infliger le châtiment qu'à ses yeux il mérite amplement:

Bien que j'eusse à bon droit de l'égorger envie,

Pour vous gratifier je luy donne la vie:

Non qu'il ne soit puny: car un si grand forfait

Ne doit couler sans peine à celuy qui l'a fait. (III, vv. 955-958)

Il compense cependant ce renoncement en redoublant de violence: il lui annonce qu'il lui fera lourdement payer son intervention en faveur du roi malheureux, qu'il se revaudra sur la caste ecclésiastique du peuple juif:

Vous vivrez vous vivrez, mais sera tellement

Que vos jours rouleront en continu tourment.

Vous requerrez la mort de borner vos tortures,

Voyant devant vous meurtrir vos creatures,

Esgorger vos amis, les prestresde la loy,

Qui mutins vous ont fait elever contre moy. (III, vv. 965-970)

Il continue d'étendre sa vengeance en ravissant les enfants du roi de Jérusalem. Et dans une fin d'apocalypse, il tente d'assouvir son dessein cruel en ordonnant une tuerie: il fait exécuter le pontife Sarrée, les princes du peuple, les enfants de Sédécie devant les yeux de leur père, et aveugler celui-ci. Or il constate qu'il ne parvient pas à s'imposer à ses victimes par sa cruauté, que celles-ci ne craignent point la mort à laquelle il les condamne, à l'instar des princes du peuple:

Le tyran eut despit en son ame bourrelle

De leur voir au martyre une asseurance telle,

Et tost se repentit de les avoir contraints

D'eschapper par la mort ses violentes mains. (V, vv. 1979-1982)

Quant à Sédécie, il ne répond pas aux accusations réitérées de Nabuchodonosor, continuant ainsi à refuser de le reconnaître comme son maitre; et ensuite bien qu'il ait assisté impuissant à l'assassinat du pontife Sarrée et de ses enfants, et qu'il ait le cœur transpercé par la douleur, il persiste dans son refus de se plier à la furie vengeresse du roi d'Assyrie se bornant à demander à ses bourreaux une fin rapide:

Puis voyant les bourreaux à la hideuse face,

Teints de sang s'approcher, humblement leur rend grace

De venir terminer par une prompte mort

L'indomtable douleur qui ses entrailles mord. (V, vv. 1995-1998)

La tentative d'anéantir son rival en lui arrachant la plupart des êtres qui lui sont chers, d'apaiser son désir métaphysique se solde donc chez Nabuchodonosor par un fiasco. 


\section{Culpabilité et innocence}

L'indifférenciation engendre la violence, qui à son tour accroît l'indifférenciation, laquelle accroît de nouveau la violence, dans une spirale toujours recommencée. Pour mettre fin à la recrudescence de la violence réciproque, est nécessaire l'unanimité de tous contre une victime qui ne peut être vengée. Comme le déclare René Girard, «le sacrifice polarise les tendances agressives sur des victimes réelles ou idéales, animées ou inanimées mais toujours non susceptibles d'être vengées, uniformément neutres ou stériles sur le plan de la vengeance. Il fournit à un appétit de violence dont la seule volonté ascétique ne peut venir à bout un exutoire partiel, certes, temporaire, mais indéfiniment renouvelable et sur l'efficacité duquel les témoignages concordants sont trop nombreux pour être négligés. Le sacrifice empêche les germes de la violence de se développer. Il aide les hommes à tenir la vengeance en respect» ${ }^{12}$.

Nabuchodonosor peut décharger sa violence sur la victime désignée: Sédécie est un étranger, c'est le roi d'un peuple esclave qui s'est révolté contre lui, qui a agi de connivence avec un ennemi héréditaire, qui a été vaincu, fait prisonnier, qui est à sa merci; last but not least, les Juifs croient à un Dieu qui n'est adoré que par eux, et que le roi d'Assyrie tourne en dérision (vv. 1385-1388). Or ce dernier ne parvient jamais à obtenir l'unanimité dans son dessein de mettre à mort le roi de Jérusalem. Nabuzardan l'invite à faire preuve de clémence à l'égard de son prisonnier:

Mais un Roy qui peut tout, n'a qu'à se retenir,

Si quelqu'un l'a fasché, de ne le trop punir.

Que de ce Roy la faute inhumain ne vous rende. (II, vv. 277-279).

Son inhumanité soulève la réaction de sa femme:

NABUCHODONOSOR La vengence tousjours un brave cœur remarque.

LA ROYNE Rien ne le souille tant qu'un fait de cruauté.

NABUCHODONOSOR Qui n'est cruel n'est pas digne de royauté.

LA ROYNE Des peuples vos sujets l'advis est au contraire. (III, vv. 908-911)

Le prévôt de l'hôtel déplore lui aussi la brutalité de son souverain:

Pleust aux Dieus immortels de n'avoir onque esté,

Plutost qu'estre réduit à ceste extremité

D’obéir aux fureurs d'un tyrannique maistre. (IV, vv. 1567-1569)

Paradoxalement, ce dessein se retourne contre lui, il fait même l'unanimité à son encontre. Le processus du bouc émissaire s'interrompt donc dès ses premières pha$\operatorname{ses}^{13}$. Ne pouvant être canalisée vers la violence purificatrice qui aboutit au sacrifice, la violence impure de la vengeance déferle, emportant tout sur son passage: Nabuchodonosor donne au prévôt de l'hôtel et à ses bourreaux l'ordre de punir lourdement,

(12) R. GIRARD, La violence et le sacré, Paris, Hachette, collection Littératures, 2008, p. 32.

(13) M.-L. Martinez indique les cinq temps du bouc émissaire: $\ll 1$ ) une crise surgit avec indifférenciation; 2) le rassemblement de tous se fait contre une victime choisie selon certains traits particuliers; 3) pour l'éviction de la victime, l'imaginaire persécuteur invente les accusations; 4) de la victime jaillissent les règles culturelles, du désordre jaillit l'ordre; 5) la victime qui était chargée de tous les maux est alors positivée voire sanctifiée». Approche anthropologique mimétique et clinique. Conférence donnée à Marseille en avril 2007 dans un contexte associatif. M.-L. Martinez est maître de conférences en science de l'éducation à l'UMR/ADEF de l'Université de Nice Sophia Antipolis. 
de mettre à mort ceux qu'il considère comme une tache à sa royauté ${ }^{14}$. René Girard s'interroge sur les dangers que comporte la vengeance du sang: «La seule vengeance satisfaisante, devant le sang versé, consiste à verser le sang du criminel. Il n'y a pas de différence nette entre l'acte que la vengeance punit et la vengeance elle-même. La vengeance se veut représaille et toute représaille appelle de nouvelles représailles» ${ }^{15}$.

A Sédécie qui essaie de comprendre pourquoi Dieu a permis la vengeance de Nabuchodonosor, le prophète fournit l'indication suivante:

Et ne sçavez-vous pas qu'il le fait tout expres,

Le souffre en ses douleurs, pour l'en punir apres?

Il use de sa dextre à venger son colère,

Comme fait d'une verge une prudente mere

Envers son cher enfant, quand une mauvaitié

Qu'il a fait à quelqu'un, veut qu'il soit chatié.

Car apres cet usage en la flamme on la rue,

$\mathrm{Ou}$ avecques mespris est en pieces rompue.

Ainsi Dieu vengera les massacres commis

Par ce Roy carnacier, bien qu'il les ait permis.

Les maux qu'il nous a faits il lui saura bien rendre,

Et quelquefois sera Babylon mise en cendre. (IV, 2113-2124)

Il affirme en substance que la vengeance revient à Dieu, oppose la vengeance de Nabuchodonosor et celle du Très-Haut. La première demeure incapable de pardon, imposée par la force, injuste. On pense à un fragment des Pensées de Pascal: juste $^{16}$.

Et ainsi ne pouvant faire que ce qui est juste fût fort, on a fait que ce qui est fort fût

René Girard établit une distinction entre l'immanence de la violence coupable et illégale et la transcendance effective de la violence sainte, légale, légitime ${ }^{17}$. La vengeance de Dieu est inspirée par le pardon, offre la possibilité de passer de l'expiation au rachat, constitue un modèle de justice ${ }^{18}$. C'est devant celle-ci que s'inclinent Sédécie et le prophète, que se confessent le chœur des Juives (vv. 485-491) le pontife Sarrée (vv. 1960-1962) et le roi de Jérusalem:

C'est pour avoir péché devant ta sainte face,

O père, \& n'avoir craint le son de ta menace (IV, vv. 1288-1289)

Et, plus loin, il ajoute:

Je scay bien que je l'ai [Dieu] mille fois irrité,

Que j'ay son ire esmeuë, \& que par mon seul crime

J'ai incité à mal toute Jerosolyme.

Je suis cause de tout, je le scay (V, vv. 2105-2109)

Il reconnaît sa culpabilité, accepte sa punition. S'il est coupable; si «la jeunesse ardante \& prompte aux changemens», qu'évoque Amital lorsqu'elle affronte Nabu-

(14) La métaphore est employée par Nabuzardan à l'acte II, v. 270: «Ne puisse donner tache à vostre Royauté».

(15) La violence et le sacré, cit., p. 28.

(16) Pensées 103-298, édition de P. Sellier, Pa- ris, Hatier, collection Thema, 1972, p. 66.

(17) Cf. La violence et le sacré, cit., p. 40.

(18) Cf. l'introduction de Sabine Lardon, cit., pp. 35-36. 
chodonosor (III, vv. 1045 sq), l'est également, et reçoit elle aussi sa sanction, les enfants que fait égorger le roi d'Assyrie présentent un cas différent. Charles Mazouer met en relief le problème que cause la punition de ces victimes innocentes ${ }^{19}$. Raymond Lebègue relève une contradiction entre les deux passages qui suivent:

SÉDÉCIE N'afflige les enfants pour le péché du père. (IV, v.1336)

LE PROPHETE Tu reçois, Israël, les rigoureux salaires

De tes propres pechez, \& de ceux de tes peres. (V, vv. 2083-2084) ${ }^{20}$

Affrontant à son tour le problème, Sabine Lardon affirme: «Le péché est celui de tout un peuple et tous sont touchés, Amital, les reines et leurs enfants, complices malgré eux des dérives d'Israël» $\gg^{21}$.

Il nous semble que l'on ne peut pas mettre sur le même plan les fautes commises par Sédécie et ceux qui l'ont suivi dans ses offenses à Dieu et dans sa rébellion contre Nabuchodonosor, et le babillage d'enfants attachés aux seins de leurs mères:

LES REINES On vous emmeine, enfans, on vous emmeine helas!

Et vous ne serez plus pendans entre nos bras

Nous baisotant le sein de caresses mignardes,

Et tirans nos cheveux de vos mains fretillardes,

Parlant vostre enfantin, \& les heures passant

Avec vos compagnons en esbat innocent. (IV, vv. 1757-1762)

Le sort de ces enfants, innocents et persécutés, fait penser à celui des Saints Innocents, ces enfants de moins de deux ans massacrés sur l'ordre d'Hérode à Jérusalem durant les premiers mois de la vie de Jésus. Leur pureté et les persécutions dont ils sont l'objet annoncent également la passion et la rédemption du Christ, lui aussi innocent et persécuté. On reconnaît là les traits des martyrs; et on reconnaît également la figure du Christ, haï sans cause ${ }^{22}$. Or l'idée du martyre est évoquée à plusieurs reprises dans le texte:

AMITAL Je vy pour le martyre (II, v. 373)

AMITAL Là donc martyrez moy, versez sur moy votre ire,

Le tourment que j'auray sera double martyre,

Torturant mere \& fils par ma seule douleur;

Sçauriez-vous inventer un outrage meilleur? (III, vv. 1086-1090)

LE PROPHÈTE Le tyran eust despit en son ame bourrelle

De leur voir au martyre une asseurance telle (V, vv. 1979-1980)

LE PROPHÈTE Hà qu'il souffre, hà qu'il souffre un angoisseux martyre. (V, v. 2092)

(19) Cf. C. Mazouer, La théologie de Garnier dans «Hippolyte» et «Les Juifves»: du destin à la Providence, dans Lectures de Robert Garnier, «Hippolyte», «Les Juifves», textes réunis par E. BurON, Rennes, Presses universitaires de Rennes, 2000, pp. 113-125.

(20) Cf. R. Lebègue, Robert Garnier, «Les Jui- fves», Paris, Centre de Documentation Universitaire, 1962 , p. 143

(21) Cf. S. LARDON, L'influence de Sénèque dans «Les Juifves» de Robert Garnier, «Studi Francesi», n. 141, anno XLVII, Fascicolo III, settembre-dicembre 2003 , pp. $527-539$, p. 538

(22) Evangile selon saint Jean, 15, 25. 
Bien que profondément meurtri dans son âme et dans son corps, Sédécie accepte ses tourments en bénissant Dieu:

SÉDÉCIE Qui vit si miserable? autour de ceste messe

Voyez-vous un malheur qui mon malheur surpasse?

LE PROPHÈTE Non, il est infini, de semblable il n'a rien.

Il en faut louer Dieu tout ainsi que d'un bien.

SÉDÉCIE Tousjours soit-il benist, \& que par trop d'angoisse

Jamais desesperé je ne le deconnoisse. (V, vv. 2099-2104)

Il n'a point cédé à la tentation de la vengeance; il a purgé son âme de la violence, de la rivalité mimétique, du désir métaphysique. Jean-Raymond Fanlo relève justement que «privé de tout, dans l'excès qui l'a fait passer de la puissance royale à une vie nue sans postérité, Sédécie accède au savoir. Il connaît quand il ne sait plus rien et met tout sons espoir en Dieu. Il se connaît quand il se perd de vue et reconnaît la toute puissance divine» ${ }^{23}$.

Il s'ouvre totalement à Dieu et est prêt à entendre la parole du prophète qui lui montre l'avenir de son peuple, et lui annonce la venue de l'agneau de Dieu, le Sauveur:

Et au temple devôt par nous redifié

Dieu mieux qu'auparavant sera glorifié,

Les autels fumeront de placables hosties,

Et seront des faux Dieux nos ames diverties.

Quelques siecles apres le Seigneur envoyra

Son Christ, qui les pechez des peuples netoyra,

Destruisant les Enfers, \& desiré Messie

Viendra pour mettre fin à toute Prophetie. (V, vv. 2165-2172)

Le Christ, ou le médiateur entre Dieu et les hommes. On pense à saint Paul:

Car Dieu ne nous a pas destinés à subir sa colère, mais à posséder le salut par notre Seigneur Jésus Christ, mort pour nous afin que, veillant ou dormant, nous vivions alors unis à luii ${ }^{24}$. lui.

Le Christ, ou la fin de toute médiation, car Dieu fait homme par amour pour

BERNARD GALLINA

(23) Cf. J.-R. FAnLO, Sentiment du tragique et piété pénitentielle dans «Les Juifves», op. cit., n. 15, Novembre 2000, pp. 43-51, p. 46.

(24) Première lettre aux thessaloniciens, 5, 9-10.

On pense aussi à Pascal: «Dieu par J.-C. Nous ne connaissons Dieu que par J.-C. Sans ce médiateur est ôtée toute communication avec Dieu. Par J.-C., nous connaissons Dieu». Pensées 189-547, cit., p. 35 . 